\title{
Electrodiagnosis in paediatric ophthalmogenetics
}

\author{
Patricia Apkarian * \\ Department of Physiology I, Faculty of Medicine, Erasmus University, P.O. Box 1738, 3000 DR Rotterdam, The Netherlands
}

(Accepted 17 January 1994)

\begin{abstract}
In the present overview, practical application of the visual evoked potential (VEP) in paediatric neuro-ophthalmology is described across a wide range of ophthalmogenetic disorders, including albinism, Pelizaeus-Merzbacher disease and spastic paraplegia. The VEP approach is based on a four parameter subdivision of the electrophysiological response which includes, (1) amplitude $(\mu \mathrm{V})$, (2) latency (ms), (3) waveform (component specificity), and (4) topography (potential distribution across the electrode array). In the case studies presented, evoked potential measures provide clinically useful and even at times invaluable insights concerning the presence, extent and type of visual pathway compromise. The four parameter subdivision along with age matched normative standards provides a sensitive and reliable means to facilitate detection and diagnosis of sensory anomalies in the developing visual system. The non-invasive electrophysiological assessment of visual function and its maturational course also provides early identification of affected family members as well as an objective measure of various treatment regimes which, particularly for the pre-verbal and non-verbal child, is critical for effective patient care.
\end{abstract}

Key words: Visual evoked potential (VEP); Development; Acuity; Defocus; Albinism; Amblyopia; Neurofibromatosis; Pelizaeus-Mer $z$ bacher disease; Spastic paraplegia

\section{Introduction}

In a previous review [1], the application of visual evoked potential (VEP) assessment in various ophthalmogenetic disorders was described. By way of a diverse sample of genetic anomalies, the review emphasized not only the importance of the VEP in the detection, differential diagnosis and prognosis in a variety of ophthalmogenetic diseases but also its importance in the specifica-

\footnotetext{
* Tel.: (+31-10) 408 7568; Fax: $(+31-10) 436$ 7594; E-mail: Apkarian@fys1.fgg.eur.nl.
}

tion of genetic patterns or modes of inheritance. In addition to its role as a diagnostic probe of functional integrity along the visual pathway, the VEP was shown to effect greater precision in the characterization of a given family pedigree. Thus, the major benefit of a non-invasive electrophysiological approach to ophthalmogenetic evaluation is more accurate genetic counselling and a concomitant improvement in treatment. If non-invasive methods for assessing visual function are applicd to the pre-verbal and non-verbal patient, a sensitive and powerful probe of visual function and dysfunction emerges. In the present overview, application of the visual evoked potential in pae- 
diatric ophthalmogenetics is described along with various stimulus and recording techniques designed to optimize VEP testing in infants and young children. An acute and poignant motive for this test approach is the need for non-invasive and accurate methods to: (1) assess the integrity of sensory function during various stages of ontogenesis, (2) detect, at an early stage, visual system anomalies that can lead to irreversible visual impairment, and (3) effect early identification of affected family members.

\section{Methodology}

\section{Paediatric stimulus and recording strategy}

For clinical purposes, the three most common modes of stimulation for recording cvoked cortical responses are the luminance flash, the pattern reversal and the pattern onset/offset or pattern appearance/disappearance, as it is also labelled (Fig. 1). At a slow rate of stimulus presentation, as depicted, each of these stimulus conditions has its own response signature in terms of number, latencies and sign (negativity or positivity) of various peaks and troughs. If the stimulus rate is sufficiently slow to allow the relevant brain responses to recover prior to the next stimulus presentation, the VEP is classified as transient.

As depicted in the luminance VEPs from a 25-week-old normal infant (Fig. 2), with higher and higher rates of stimulation, waveshape specificity deteriorates and the responses begin to resemble a sinewave with the fundamental frequency or harmonics of the stimulus frequency. If the rate of stimulation is sufficiently high to preclude response recovery, the VEP is termed stcady state. Both the higher frequency steady state VEP and the transient VEP are important for clinical application and frequently yield complementary information. The latter, however, also yield waveform information which, as seen in Figs. 3 and 4 , is particularly useful in assessing response maturation. As illustrated for both the luminance flash and the pattern onset, the response profiles, including waveform, amplitude and latency are age sensitive. The development of characteristic
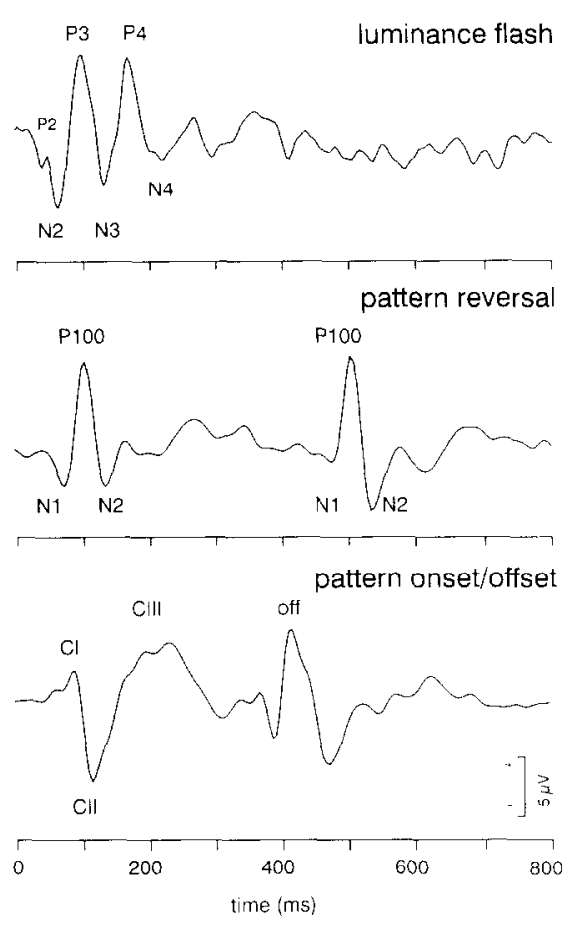

Fig. 1. Three classic VEP types recorded from an adult to luminance flash (upper trace), pattern reversal (centre trace) and pattern onset/offset (lower trace) stimulation. The luminance flash (recorded with $0.6 \mathrm{~J}$ light intensity) is a complicated waveform consisting of several major and minor positive and negative response deflections. The pattern reversal response (recorded with a $96^{\prime}$ check size and with each reversal presented once every $400 \mathrm{~ms}$ ) consists of a negative-positivenegative waveform complex. The pattern onset/offset response (recorded with an $18^{\prime}$ check size) consists of a triplasic onset featuring a positive-negative-positive waveform complex and an offset consisting primarily of a single positive peak. In this example, the pattern was presented for $300 \mathrm{~ms}$ and was replaced with a homogeneous field of the same mean luminance for $500 \mathrm{~ms}$. Mean luminance and contrast for both pattern responses was $95 \mathrm{~cd} / \mathrm{m}^{2}$ and $80 \%$, respectively (adapted from [3]).

adult-like responses, particularly in waveform, requires a rather lengthy maturational course.

\section{Visual stimulation}

The two stimulus configurations most frequently referred to in this overview are the checkerboard (primarily pattern onset), and homogeneous luminance field (primarily luminance 
flash). Checkerboards, typically generated on a computer driven monitor, are the pattern configuration of choice over more simple patterns such as horizontal or vertical stripes. The rational behind this choice is two-fold. First, because of greater contour (edge) information, checkerboards typically yield larger amplitude responses than corresponding bar patterns [2]. Second, the checkerboard, with its multiple contour orientations may more readily bypass stimulation orientation deficits due to nystagmus and/or refractive errors such as astigmatism $[3,4]$. In terms of mode of pattern stimulation, the pattern onset is the method of choice. The rational behind this preference is also two-fold. First, the pattern onset, particularly the negative CII deflection, reflects primarily spatial contrast responses compared to the stimulus reversal mode which, in addition, also reflects stimulus motion responses [5-7]. Pattern reversal contamination of a pattern spe- cific response with a movement response is most likely the reason that the pattern onset (unlike the pattern reversal) correlates more directly with behavioural estimates of spatial vision. Second, the pattern onset stimulus is more appropriate for patients with oculomotor instabilities and/or misalignments and for younger patients since the lengthy and highly accurate fixation demands of the pattern reversal are not required for pattern onset $[3,4,8]$.

Unless otherwise noted, for data presented herein, the contrast of the check elements was $80 \%$; mean luminance of the stimulus field was around $100 \mathrm{~cd} / \mathrm{m}^{2}$.

Luminance flash stimulation was typically generated with a commercially available light flasher which allows high intensity pulses of light (a $1 \mathrm{~Hz}$ rate and $0.6 \mathrm{~J}$ intensity were employed in this study). However, a homogeneous luminance stimulus, sinewave modulated, is an appropriate stim-

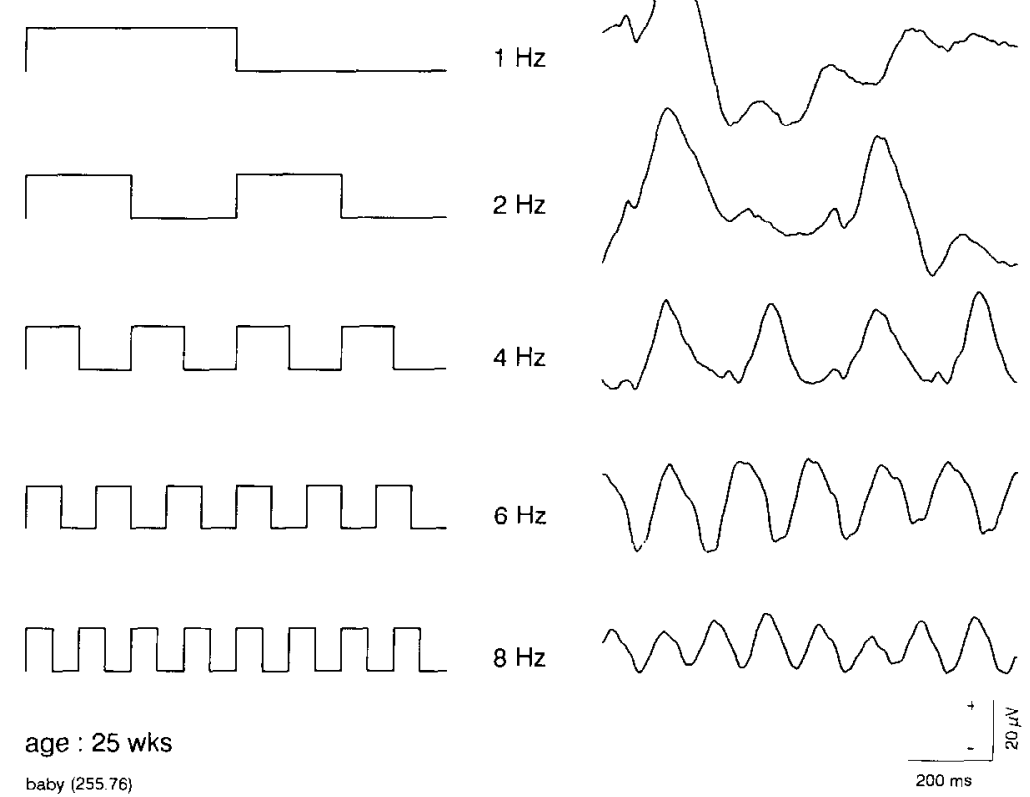

Fig. 2. Transition from transient to steady state VEP with increasing stimulus frequency. The luminance flash VEPs depicted (right traces) were recorded from a 25 -week-old full term infant to square wave modulation of a light source (left traces). Responses at 1 $\mathrm{Hz}$ and $2 \mathrm{~Hz}$ show the expected waveform complexes. As the frequency rate increases, specific waveform features diminish and the response develops a sine-wave appearance with a fundamental frequency equal to that of the stimulus frequency (adapted from [3]). 
ulus configuration for assessing temporal vision (i.e., temporal responsivity). Growth functions of VEP temporal responsivity are now available [9].

\section{VEP recording}

To record pattern or luminance responses, an appropriate recording montage is important. For the data presented, the electrode montage consisted of five "active" electrodes positioned across the occiput, $1 \mathrm{~cm}$ above the inion and linked to a high forehead reference (or linked ear lobes). An additional ground was placed at the vertex. Filtering and amplification followed standard VEP recording procedures. For more details on stimulus and recording procedures in paediatric populations, see Apkarian (1994).

MATURATION: LUMINANCE FLASH
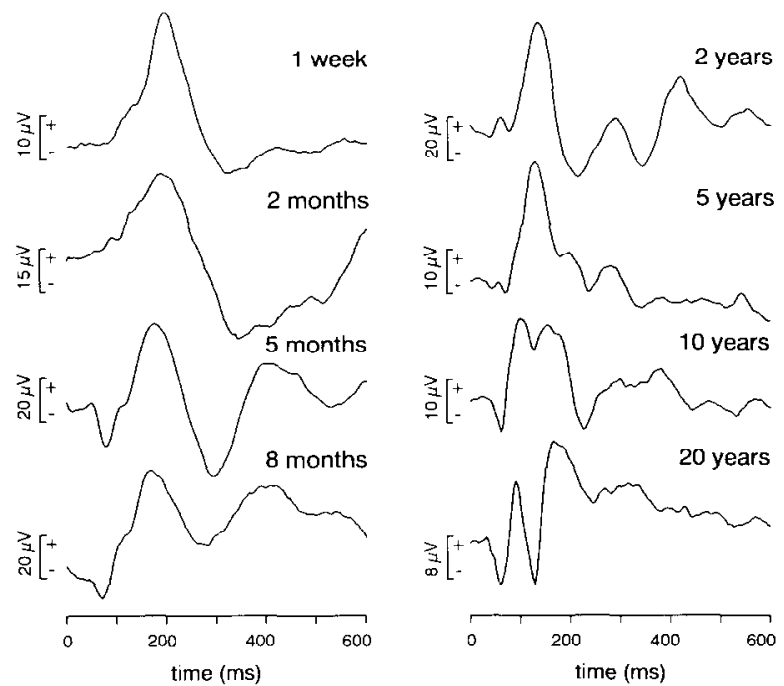

Fig. 3. Maturation of the luminance flash VEP from 1 week of age to 20 years. Within the first months of life the luminance flash response is simpler in waveform and is characterized by a major positive peak with latency of about $200 \mathrm{~ms}$. During development, the luminance flash response increases in complexity and the latency of the major deflections decreases. In the adult the first major positive peak has a latency around $100 \mathrm{~ms}$. Very early latency deflections appear less sensitive to maturational changes. Note also the trend towards an inverted U-shaped function of response amplitude as a function of age (adapted from [3]).

\section{MATURATION: PATTERN ONSET 12'}
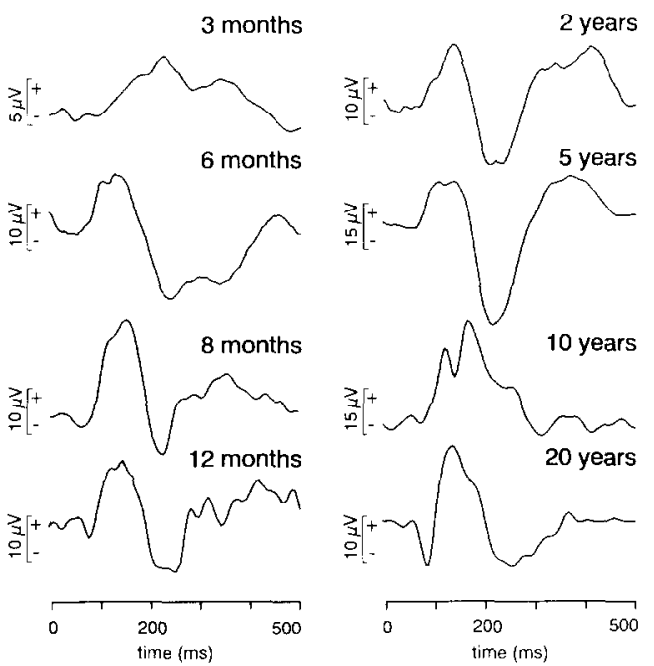

Fig. 4. Maturation of the pattern onset VEP from 3 months of age to 20 years. The immature pattern onset response consists primarily of a single, slightly sluggish, positive waveform denection with a latency, after about 3 months, of about $150 \mathrm{~ms}$. The major maturational improvements occur primarily in response waveform which reaches the adult-like positive (CI), negative (CII), positive (CIII) waveform complex around $\mathrm{pu}^{-}$ berty. Note that at around 10 years of age, identification of response components is difficult as the response begins to reflect a transitional stage. Pattern onset was $40 \mathrm{~ms}$, offset 460 $\mathrm{ms}$, pattern size $12^{\prime}$, mean luminance $90 \mathrm{~cd} / \mathrm{m}^{2}$ and contrast $80 \%$ (adapted from [3]).

Lastly, if neonates and younger infants are tested, it is important to record the visual evoked responses only under behavioral state defined conditions $[9,10]$.

\section{Results and discussion}

In addition to emphasizing maturational changes in the VEP response profile, the approach adopted in this overview of electrodiagnosis in paediatric ophthalmogenetics is rather straightforward.

Response analysis concentrates upon four simple response parameters: amplitude $(\mu \mathrm{V})$, latency (ms), waveform (component specificity) and to- 
pography (potential distribution across electrode array). The four-parameter subdivision of the evoked response is purely descriptive in that these response variables are largely interdependent. With this basic approach in mind then, what can be expected from examination of the first VEP parameter of interest, i.e., amplitude?

\section{Amplitude}

In general, one can expect an amplitude range of the transient VEP from 5 to $20 \mu \mathrm{V}$. However, perfectly normal controls may exceed this range in either direction. Further, absolute amplitude changes with age, showing in inverted U-shaped function across the age span. Because of the extreme variation of normal amplitude values even within the same age group, absolute amplitude measures for comparisons between individuals typically are not clinically viable.

However, in some clinical conditions absolute amplitude values may be used to establish presence or absence of measurable responses. Application of absolute amplitude is shown in Fig. 5, depicting responses from an infant born with severe and multiple congenital malformations. The primary clinical question in this casc was simply, "Is this baby blind? Can she see light; can she see patterns?" As can be seen from Session I, for this infant VEP signals of sufficient amplitude to be distinguished from background noise yield a positive answer to both of these questions and in this case also contributed to the medical decision to provide this infant with life support surgery.

If more specific questions are posed such as, "How much can the baby see and/or are there maturational improvements?" then relative amplitude measures within the same individual provide more information. In Fig. 5, Session II, for example, the minimum and optimum pattern sizes which yield reliable responses demonstrate maturational improvements. Relative amplitude measures can also provide useful information regarding visual acuity. How is this accomplished?

This query is addressed by the following example which stresses the close relationship between the smallest pattern size yielding a VEP response and Snellen acuity. At the left of Fig. 6 are presented the binocular pattern onset VEPs from
CRANIOSTENOSIS AND MICROPHTHALMUS

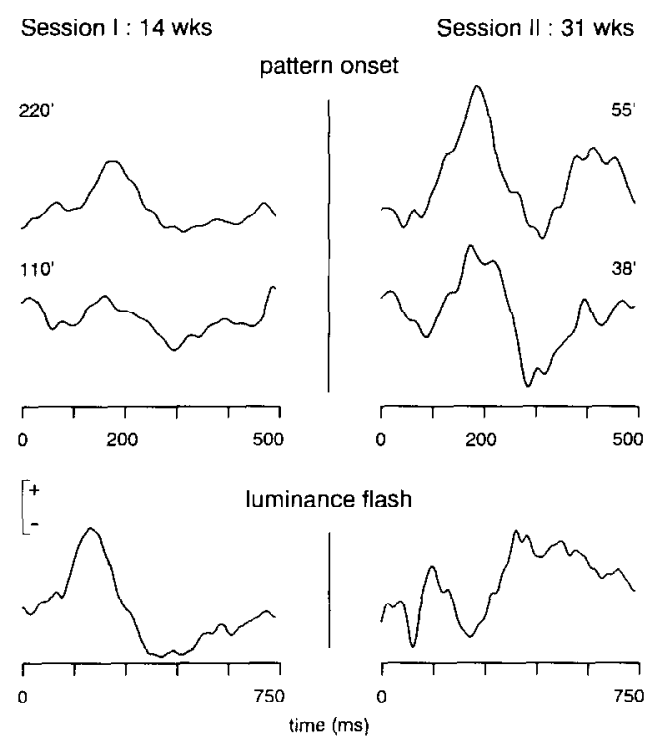

Fig. 5. VEP amplitude measures to determine presence or abscnce of a visual response and to monitor development in an infant with craniostenosis, microphthalmus and other congenital anomalies. For Session I (left), both a reliable pattern onset response and a response to luminance flash were present. The pattern size yielding the largest amplitude was $220^{\prime}$, the smallest, $110^{\prime}$. During follow-up approximately 4 months later (Session II), measurable improvement in visual responsivity was noted. The pattern size yjelding the largest amplitude improved to $55^{\prime}$ and the minimum decreased to $38^{\prime}$. Note, also, that both the pattern and luminance flash responses are poorly differentiated and show, particularly for Session II, delayed response latencies for age. Vertical calibration bar equals $5 \mu \mathrm{V}$ for pattern onset responses and 10 $\mu \mathrm{V}$ for luminance flash (adapted from [3]).

a normal 8-year-old. Notice that the pattern onset response is immature. Rather than a tri-phasic waveform with clearly distinct $\mathrm{Cl}$ (positive), CII (negative) and CIII (positive) deflections, the responses are dominated by a single positive peak labelled in this overview as PI; the longer latency negative deflection is labelled N1. With smaller and smaller pattern sizes, VEP amplitude decreases. To the right, various parameters of the responses have been quantified in relation to pattern size. For example, in the upper graphs, amplitude (of P1) and latency (of P1) have been 
plotted as a function of pattern size while in the lower graph, amplitude at varying pattern sizes is plotted as a function of electrode from left to right occiput. For practical purposes, VEP acuity can be estimated simply by determining the smallest pattern size yielding a reliable response $[11,12]$. Under the test conditions of the data presented here, a pattern size of $3^{\prime}$ yields a Snellen equivalent of $1(20 / 20)$. The correlation diagram of Fig. 7 shows that with appropriate recording conditions, there is a significant and linear correlation between VEP acuity and Snellen acuity.
With this extraordinarily simple yet powerful means of determining visual acuity, it is possible to assess, in Snellen equivalents, spatial vision objectively. This is of particular importance for the pre-verbal and non-verbal patient. Fig. 8 depicts the VEP response profile from a severely psychomotor retarded child. Psychomotor retardation is of frequent incidence in a variety of genetic disorders [13]. Despite the serious behavioral limitations with which this child presents, relative VEP measures allow not only the determination of visual acuity but also the objective assessment of visual maturation and the detection

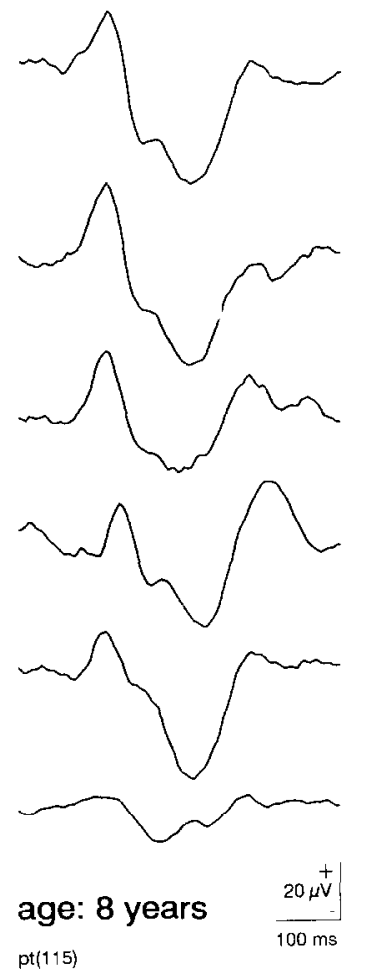

$110^{\prime}$

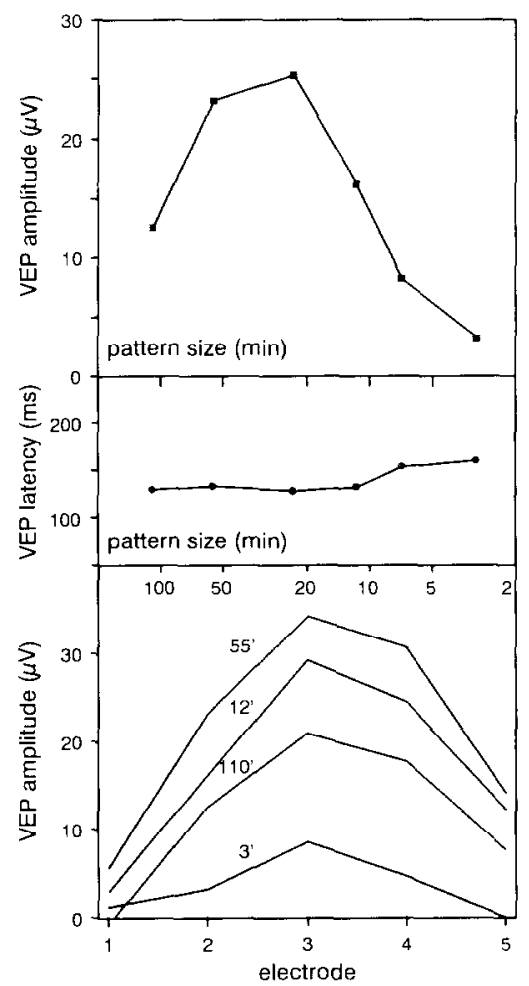

Fig. 6. VEP measures of spatial vision for acuity assessment. Left column depicts binocular pattern onset responses from a normal 8-year-old control to varying pattern sizes. The immature response consists of primarily a single positive peak which increases in amplitude in response to the optimum pattern size and then decreases in amplitude as pattern size decreases as shown quantitatively in the amplitude versus pattern size function (upper right) plotted for an occipital electrode positioned one cm above the inion and three $\mathrm{cm}$ left of midline (electrode no. 2). Peak amplitude values for the data depicted are measured peak to baseline (mean amplitude of the first $40 \mathrm{~ms}$ of the response). The smallest pattern size yielding a response provides the VEP acuity. In this case the smallest pattern size was about $3^{\prime}$, yielding a VEP acuity in Snellen equivalents of $1(20 / 20)$. VEP latency is also affected by pattern size showing an inverse relationship as depicted in the latency versus pattern size function (centre insert). The effect of amplitude on electrode position across varying pattern sizes is illustrated in the amplitude versus electrode function (lower right). In this example, the peak amplitude responses occur at the midline (occipital electrode no. 3 ). 
of possible interocular differences. In this case, VEP amplitude measures show an interocular difference of nearly fourfold supporting the ophthalmic indication of amblyopia. An additional point of interest is that while pattern responses show an abnormal interocular amplitude difference, interocular latency is comparable for measurable responses. The amblyopic eye does just about as well as the fellow eye in terms of response latency. The relatively normal latency values for age and stimulus conditions indicate that there is no visual pathway pathology associated with delayed neuronal conduction times. What might be expected, however, in patients in which this is indeed the case?

\section{Latency}

To address this problem, the next electrophysiological variable to be examined is latency. Unlike amplitude, absolute latency values can be readily compared across subjects provided stimulus and recording conditions as well as age are carefully matched. As suggested earlier, developmental changes in latency are dramatic and certainly must be borne in mind. In the following examples, the clinical application of relative and absolute latency measures is evaluated.

Fig. 9 emphasizes absolute latency delays involving both eyes as depicted in the pattern onset response of a three year old boy. This young boy is retarded and has a serious degenerative disease called spastic paraplegia. It is a progressive, degenerative disease of primarily the spinal cord. Frequently in patients with spastic paraplegia, there is optic atrophy and visual impairment. In this child, however, ophthalmic as well as physical symptoms of spastic paraplegia were not yet present. VEP evaluation, none-the-less, revealed significantly delayed response latencies. Peak response latencies of at least $250 \mathrm{~ms}$ were recorded; for age a latency of around $150 \mathrm{~ms}$ is expected. The significantly delayed latency results indicate serious involvement of the visual pathway and in this particular case, revealed the condition of optic neuropathy prior to clinical symptoms.

Fig. 10 is presented simply to illustrate that latency delays also can occur between the two

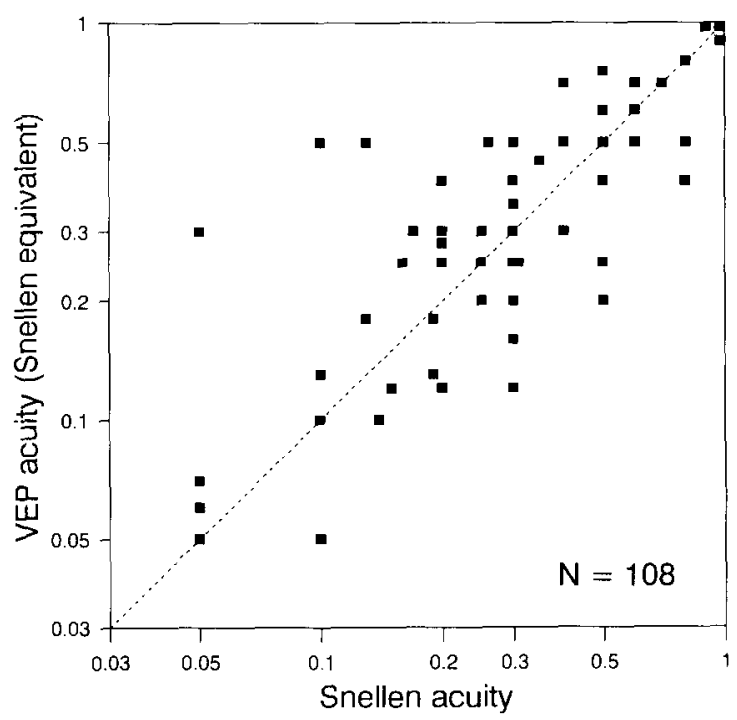

Fig. 7. Correlation diagram of VEP acuity estimates from pattern onset responses versus Snellen acuity. Linear regression analysis yields a highly significant correlation between the two variables as emphasized by the dashed line positioned along the diagonal; the regression coefficient is beta $=0.88$, $r=0.91$. Note that the analysis is based on 108 acuity correlations. The reduced number of data points illustrated is due to graphics overlap from equal values (adapted from [3]).

eyes or across the left and right hemispheres. In either case, under normal conditions, interocular or interhemispheric latencies should be about equal. In this one year old boy, however, who suffers from a tumour behind his left eye this is not the case. Responses from the left occiput from the left eye are significantly delayed compared to right occiput responses from either eye. These results indicate that the delays are due to involvement of temporal retinal projections from the left eye. In this case, the latency parameter facilitated in locating the site of visual pathway pathology.

\section{Waveform}

The previous clinical cases emphasized the VEP parameters amplitude and latency; the next electrophysiology parameter to receive attention is waveform. In examining VEP waveform, two 
questions can be posed, 1) Is the waveshape appropriate for the specific stimulus and recording conditions? and 2) is the waveshape normal for age. Both questions are addressed in the cases which follow.

The VEP response profile in Fig. 11 was recorded from a young child with a congenital malformation syndrome which obstructs normal visual input. In addition to several surgical procedures to clear the obstructing eye lids, this child also underwent occlusion therapy for possible deprivation amblyopia of the right eye. Traces to the left depict the pattern onset response; to the right are the luminance flash responses. Inspection of the responses reveals that waveforms for both the left and right eye are poorly differentiated regardless of stimulus condition.

Furthermore, no improvement is seen either with age or with occlusion therapy. Despite early surgical intervention and preventative amblyopia treatment, the VEP continued to show significant abnormalities that could not be accounted for by the condition of amblyopia alone. In this case the VEP not only provided an objective monitor of treatment but also aided in the detection of further underlying disease process.

The last case in which waveform is emphasized is presented in Fig. 12 in which the amplitude of the contrast sensitive components of the pattern onset response [11], measured as the difference in

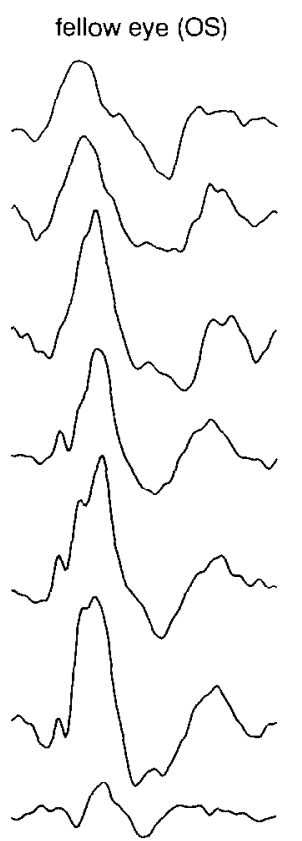

age: 6 years amblyopic eye $(O D)$

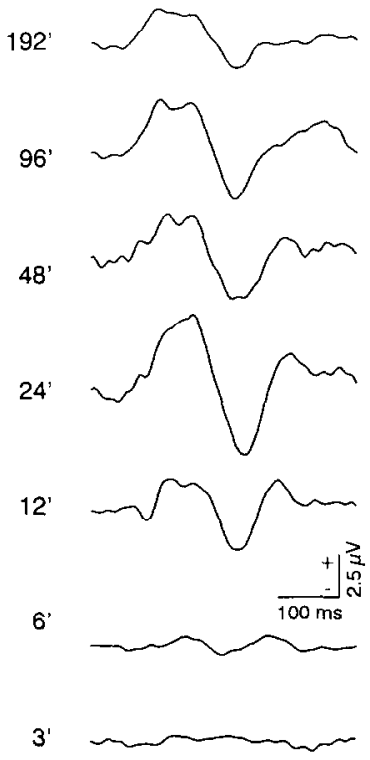

$\mathrm{pt}(300.1)$

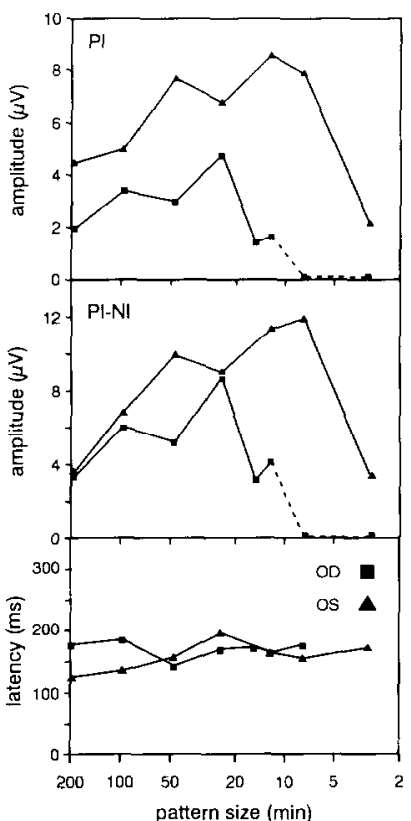

pattern size (min)

Fig. 8. VEP acuity estimated in the fellow eye (left column) and amblyopic eye (right column) of a 6-year-old with anisometropic amblyopia. Relative amplitude measures of the pattern onset response between the two eyes show that the minimum recordable pattern size differs by a factor of about 4 between the fellow eye (OS) and the amblyopic eye (OD). The peak (labelled PI for convenience) to baseline amplitude versus pattern size function (upper insert) shows an interocular difference across the pattern size range which increases as pattern size decreases. Plotting the difference amplitude between the peak and the subsequent negative deflection (PI-NI) emphasizes an attenuation of the amblyopic eye for primarily the smaller patterns and robust responses for the range of large pattern sizes (centre insert). Dashed lines for non-measurable responses are extrapolations to zero amplitude. Despite the significant amplitude attenuation of the amblyopic eye compared to the fellow eye, the monocular latency values (lower inset) are similar for measurable responses. 
amplitude of the CI-CII components, is plotted as a function of dioptric power of concave ophthalmic lenses. In this example, check size was $12^{\prime}$. It is well established that pattern specific components of the VEP response are quite sensitive to defocus and that electrophysiological measures of blur correlate well with subjective measures [14-16]. Under optimum conditions, VEP attenuation of the pattern specific response can occur with as little as 0.25 dioptres of refractive error [17]. This high degree of VEP sensitivity to optical defocus provides an objective means to measure amplitude of accommodation. Objective estimates in this case were critical as the young patient was a malingerer. Following correction of refractive error (a spherical correction of -1.75 $\mathrm{D}$ was necessary), VEP amplitude remained high and nearly constant to about $-4 \mathrm{D}$, the highest minus lens introduced. Although accommodating appropriately to the small check elements, the patient stated that no checks or patterns of any type could be seen. However, in this case objective estimates of refraction and accommodation confirmed that the discrepancy between subjective report and negative ophthalmic findings was functional rather than organic. Following VEP

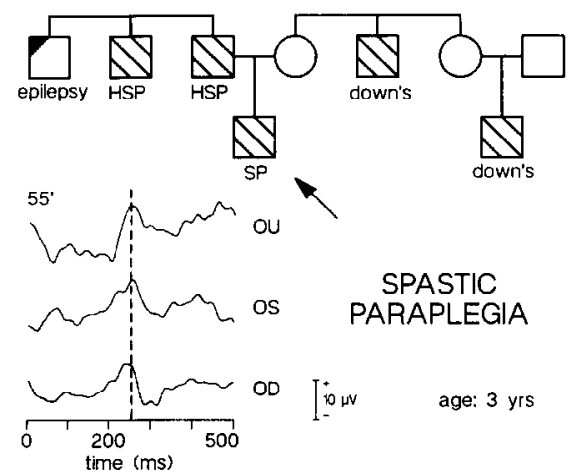

Fig. 9. Pre-symptomatic delayed latency in the binocular (OU) and monocular (OS and OD) pattern onset VEPs of a young child with spastic paraplegia. The vertical dashed line drawn through the major positive peak is severely delayed (about 250 ms) indicating optic neuropathy. The pedigree shows a dominant mode of inheritance. The proband is denoted SP (adapted from [1]).

\section{NEUROFIBROMATOSIS}
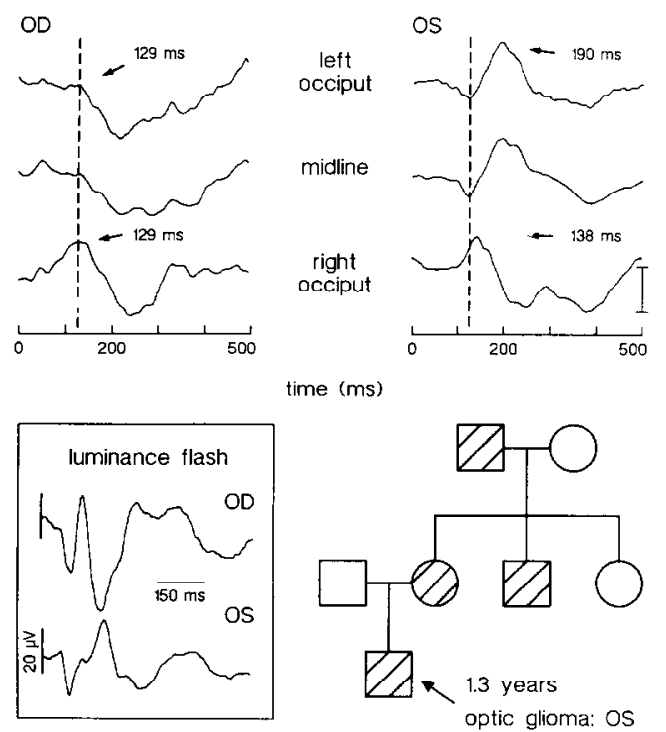

Fig. 10. Interocular and interhemispheric latency delays in the pattern onset (pattern size $55 \mathrm{~min}$ ) and luminance flash VEPs of a child with neurofibromatosis and a concomitant left eye optic glioma. The vertical dashed line in the monocular pattern VEPs derived from left (upper traces), midline (middle traces) and right (lower traces) occiput represents the peak latency $(129 \mathrm{~ms})$ for the right eye (OD). OD latency is normal for age. Abnormal, however, is the interocular latency difference (OS $>O D$ ) and a interhemispheric latency difference with the peak latency from left occipital derivation $(190 \mathrm{~ms})$ significantly longer than the right $(138 \mathrm{~ms})$. For OD the interhemispheric difference is pronounced only in terms of amplitude. Vertical calibration for the pattern responses is $10 \mu \mathrm{V}$. Midline derivations for the luminance flash VEPs (lower left) also show incongruence in latency OD compared to OS. The pedigree shows that inheritance is dominant and maternal; the proband is denoted by the arrow (adapted from [1]).

test results, the young patient was referred for psychotherapy.

Before turning to the last VEP parameter of interest, a VEP profile is presented from an infant with a rare disease effecting dysmyelination (Fig. 13). The purpose of this example is to emphasize the interdependence of the VEP variables that have been discussed. In this case, both the minimum and optimum binocular pattern on- 


\section{BLEPHAROPHIMOSIS, PTOSIS, EPICANTHUS INVERTUS}

PRE-OPERATIVE

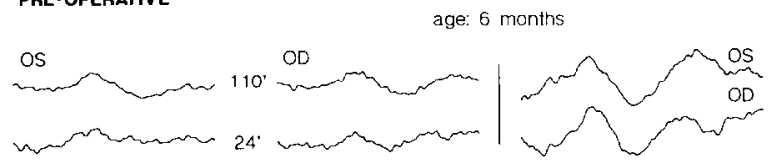

POST-OPERATIVE

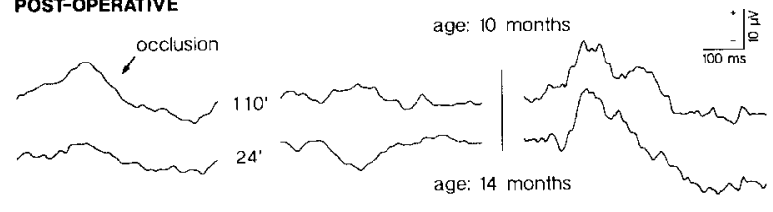
age: 14 months

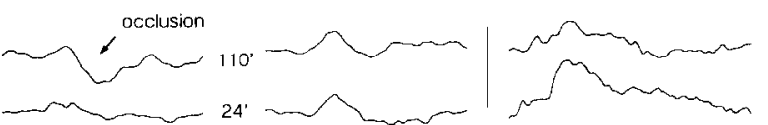

Fig. 11. Pre- and post-therapy examination of component specificity in the monocular patten onset (left traces) and luminance VEPs (right traces) from an infant with blepharophimosis, ptosis, epicanthus invertus and deprivation amblyopia shows no developmental and/or treatment improvement of VEP waveform (adapted from [1]).

set VEPs indicate delayed spatial vision. In addition both the pattcrn and luminance responses show poor waveform differentiation and delayed latencies. These results facilitated early diagnosis of Pelizaeus-Merzbacher disease (PMD), while follow-up provided an objective assessment of visual maturation and disease progression.

\section{Topography}

The final evoked potential parameter of interest is, topography. What is meant by topography is simply the inspection of the VEP responses across the electrode array, i.e., the potential distribution across the scalp. It is now well established $[1,18]$ that VEP topography across even a simple array of five occipital electrodes, shows remarkable intersubject variability and can alter from midline dominance, or midline attenuation to left or right hemispheric response dominance. Thus absolute response asymmetry per se may not be indicative of underlying visual pathway pathology. However, relative VEP topography measures have proven invaluable for disease de- tection and differential diagnosis. The case in point is albinism. The genetic anomaly in albinism precludes normal visual pathway development [19]. In albinism, temporal retinal fibres erroneously decussate at the optic chiasm disrupting functional and anatomic organization throughout the pathway [20]. The misrouting of temporal retinal fibres can be readily recorded from the surface of the scalp in the form of contralateral hemispheric response lateralization following full field monocular stimulation [21]. In Fig. 14, the traces depicted are the monocular responses from left to right hemisphere and the bottom-most traces are difference potentials between the two hemispheres. It is of importance that following monocular stimulation the potential distribution remains constant for the agematched normal control whereas for the albino, contralateral asymmetry is reflected in the poten-

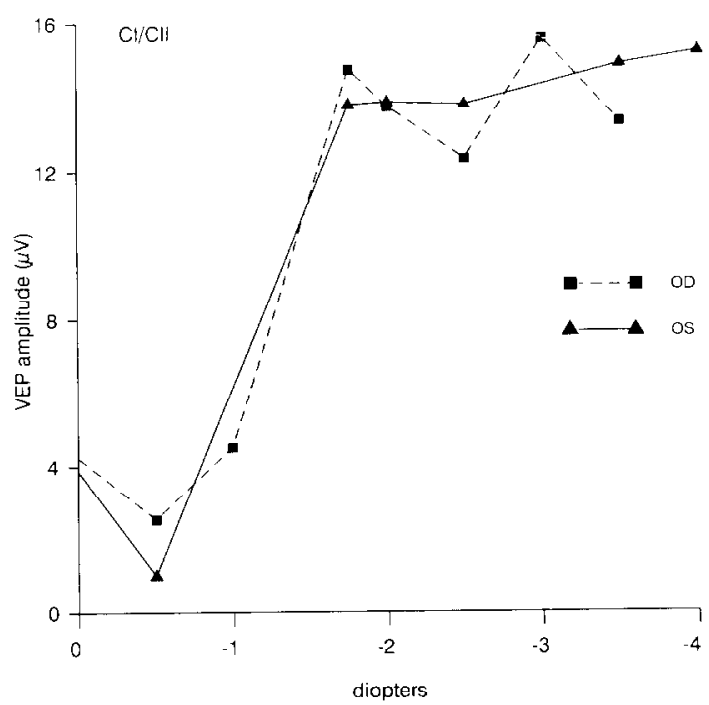

Fig. 12. VEP assessment of amplitude of accommodation in a young malingerer. Relative VEP amplitude of the CI component (amplitude of CI-CII) for both the right (stippled lines) and left (solid lines) eyes is plotted as a function of the dioptric power of concave ophthalmic lenses positioned in a trial frame before the eyes. Following spherical correction of $-1.75 \mathrm{D}$, the VEP amplitude remains high and nearly constant to about $-4 \mathrm{D}$. Check size was $12^{\prime}$, viewing distance 228 $\mathrm{cm}$ and field size $6.5^{\circ}$. 
tial distributions, in the polarity reversal of the difference potentials and in the cross-over of the amplitude versus electrode functions. For the topography graphs (to the right) VEP amplitude at the designated response latency is plotted as a function of electrode from left to right occiput for left (closed symbols) and right (open symbols) eye. For each function, a lateralization value is ascribed which defines where across the electrode array the potential distribution shows its greatest activity. Once a lateralization value per eye is obtained, an interocular asymmetry index is ascribed in which the lateralization value of the right eye is subtracted from that of the left. The rectangular bars at the right of each topography plot indicate the calculated asymmetry index of each data set. Significant interocular asymmetry is present only for the albino.
The contralateral hemispheric shift in the potential distribution from left to right eye stimulation constitutes the classic albino VEP signature. Figs. 15 and 16 show that regardless of genotype or phenotype or age, all albinos have abnormal visual pathways with VEP profiles reflecting the cross-over signature of misrouted retinal-fugal projections. As depicted, the interocular VEP asymmetry paradigm can be used to describe the family pedigree. Under appropriate age dependent test conditions, the albino VEP test can even detect misrouting in the albino neonate [10].

While we typically think of albinos as being fair haired and fair skinned, the inborn error of metabolism may effect varying degrees of dermal and ocular hypopigmentation. In addition to varying genotypes and phenotypes, it is also important to consider that, with the exception of mis-

\section{$X$-CHROMOSOMAL PELIZAEUS-MERZBACHER DISEASE (PMD)}

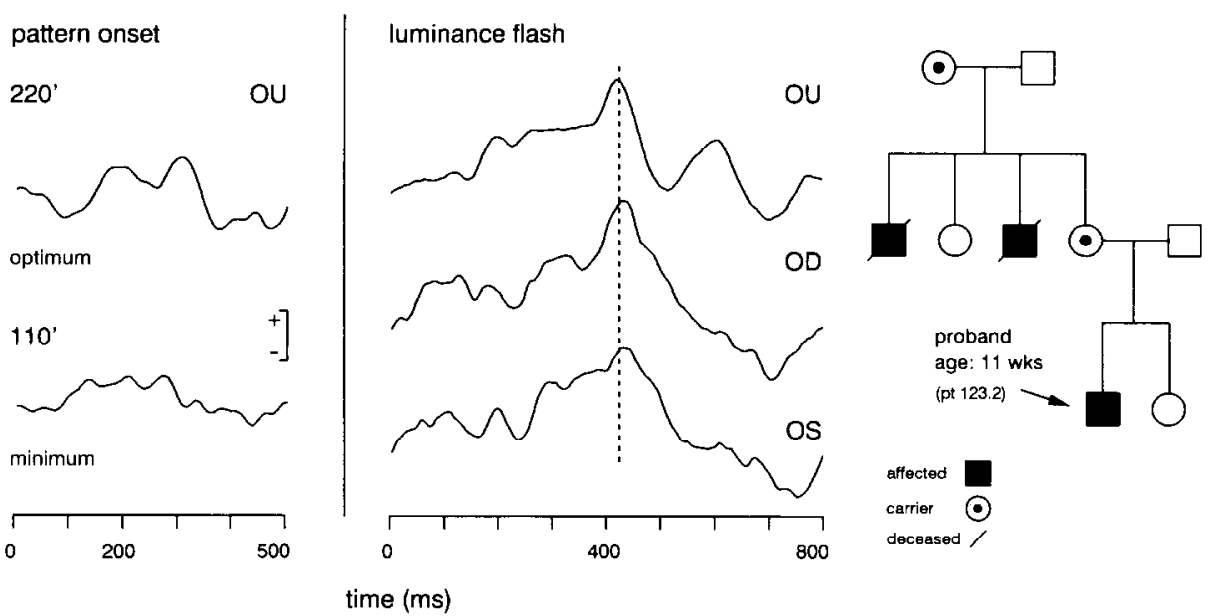

Fig. 13. Delayed latency in the minimum and optimum binocular (OU) pattern onset VEPs and the binocular and monocular luminance flash VEPs from an 11-week-old full term infant with an X-linked form of Pelizaeus-Merzbacher disease. Latency in this progressive dysmyelinating disorder is delayed for the minimum and optimum binocular (OU) pattern onset responses and for the binocular and monocular luminance flash responses. The pedigree (right) shows the mode of inheritance and afflicted family members (slashed squares). Note that two afflicted family members are deceased (crosses). The proband whose data are depicted is denoted by the arrow; round symbols represent female, squares male. The vertical dashed line drawn through the major positive peak of the luminance flash traces shows severe delay, though poor waveform differentiation of both the luminance and pattern onset VEPs renders component identification difficult. Both stimulus modes, however, yield reliable though sluggish responses and confirm the presence of pattern and luminance processing. Vertical calibration bar equals $5 \mu \mathrm{V}$ for pattern onset responses and 10 $\mu \mathrm{V}$ for luminance flash. 

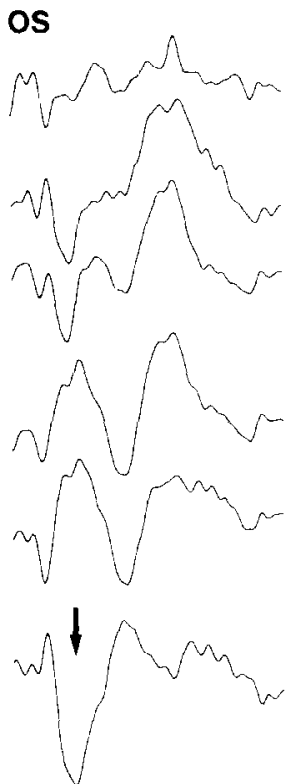

os

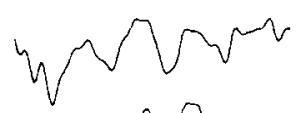

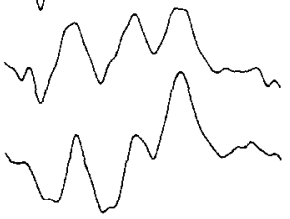<smiles>CCCCCCCCCCCCCCC</smiles><smiles>CCCCCCCCCCCCCCCCCC</smiles>

R

$O D$

$\mathbf{L}$

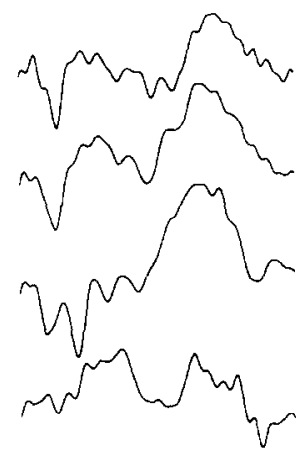

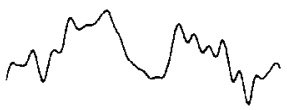

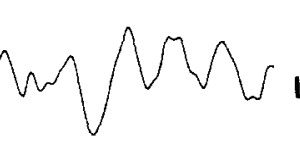

$O D$

$L$

R

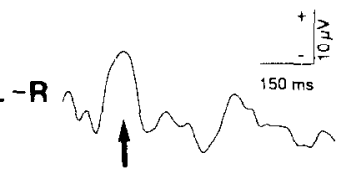

OS $188 \mathrm{~ms}$ OD $172 \mathrm{~ms}$

ALBINO PROBAND

age: 33 wks

p\#: III.3

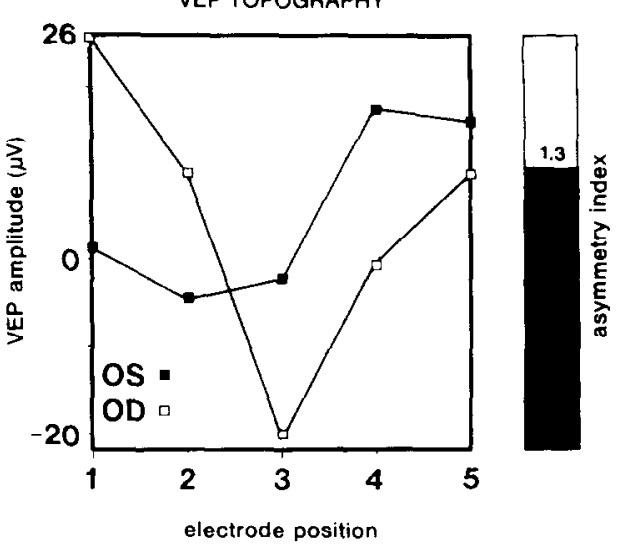

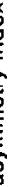

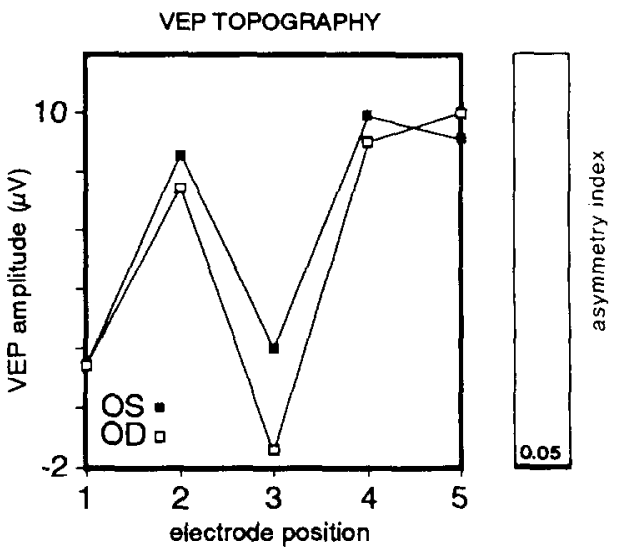

OS $188 \mathrm{~ms}$ OD $184 \mathrm{~ms}$

NORMAL CONTROL

age: 33 wks

p\#: 256.57

Fig. 14. Visual evoked potential assessment of infant proband (upper), compared with an age-matched normal control infant. Visual evoked potential contralateral asymmetry following full-field monocular luminance flash stimulation detected the condition of optic pathway misrouting in the 33-week-old patient, indicating albinism. Upper five traces are derived from electrodes positioned from the left $(L)$ to right $(R)$ occiput. Bottom traces are difference potentials obtained by subtracting trace $4(\mathrm{R})$ from trace 2 (L.). For the albino proband, with left cyc (OS) stimulation, a major positive peak of the immature luminance flash $V E P$ lateralizes to the right hemisphere; with right eye (OD) stimulation, the response lateralizes to the left hemisphere. Note the polarity reversal of the difference potentials (arrows), the cross-over of the monocular response amplitudes plotted across the electrode array and the higher asymmetry index. Compare these results with age-matched normal control. Topography plots are derived from amplitude values across the electrode array at the denoted latencies (adapted from [22]. 
routing, several non-albinos may share one or more albino clinical features, including skin hypopigmentation, nystagmus, iris diaphany, visual loss. Moreover, with the exception of misrouting and foveal hypoplasia, one or more clinical fcatures may be absent in a given albino. Thus the

\section{AUTOSOMAL RECESSIVE OCULOCUTANEOUS ALBINISM}
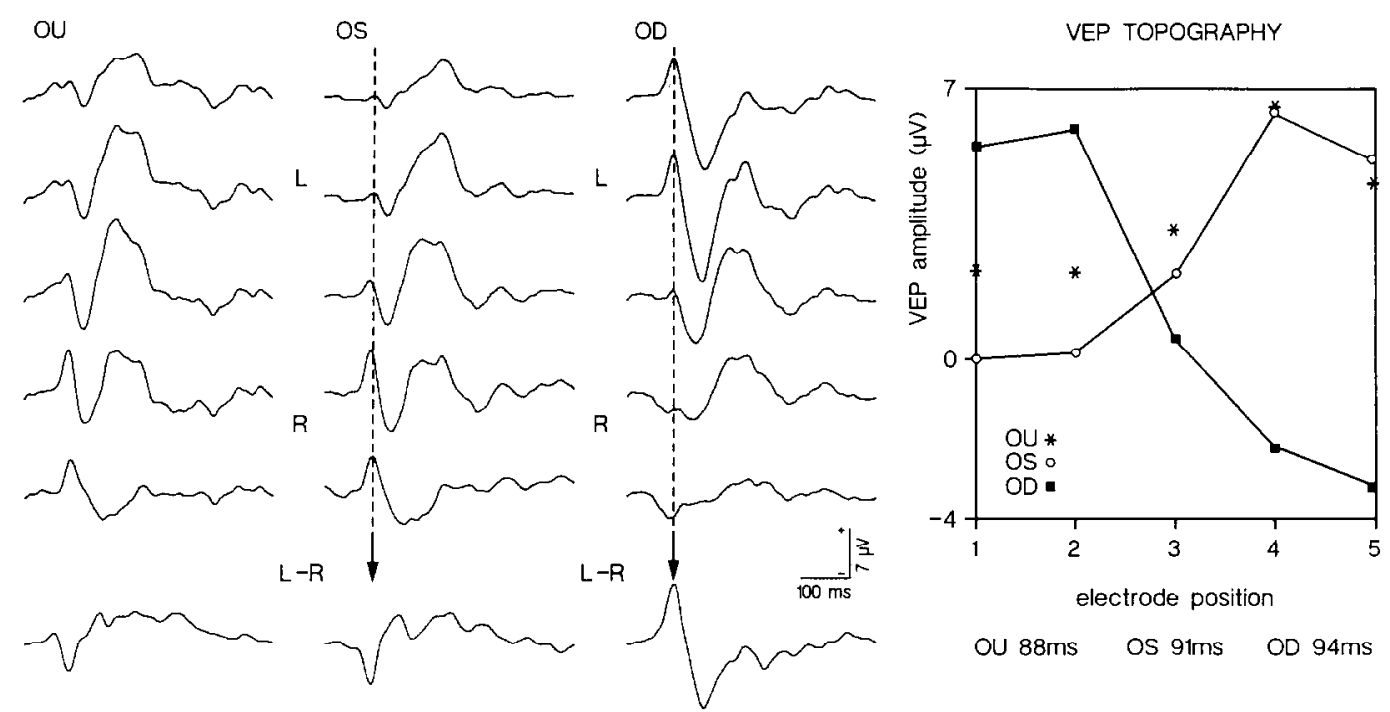

\section{OCULOCUTANEOUS ALBINO PROBAND}

flash

os

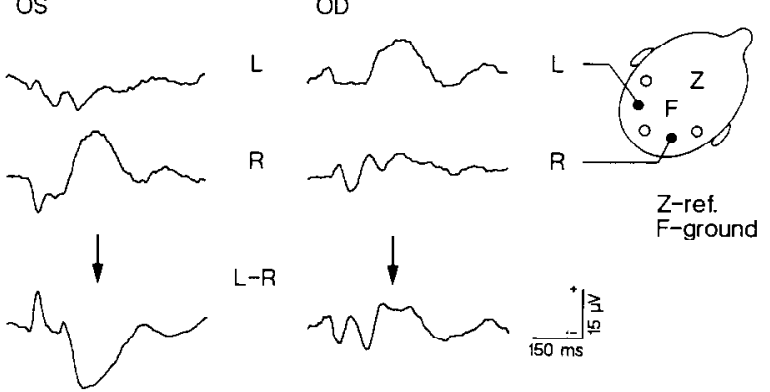

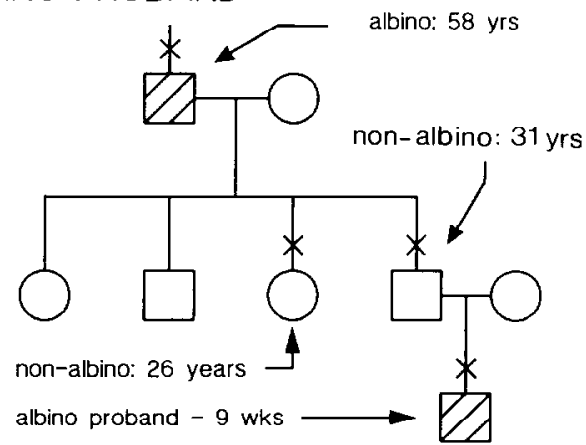

Fig. 15. Albino asymmetry in pattern onset VEPs of an adult albino (age 58 years, upper traces) and in the luminance flash VEPs of an albino infant (age 9 weeks). For more details see Fig. 14. Only traces 2 and 4 and the bipolar derivations are presented for the 9-week-old proband. For both albinos, the location of the peak of the potential distribution changes with left (OS) to right (OD) eye stimulation. The binocular (OU) VEPs from the older albino also show right hemispheric response dominance. Note, however, the cross-over of the monocular response amplitudes across the electrode array. The partial family pedigree shows autosomal recessive inheritance. The 9-week-old proband is denoted by the horizontal arrow. The albino grandfather of the proband whose data are presented above is indicated at the top left of the pedigree. Family members tested are indicated by an $X$ (adapted from [1]). 
X-CHROMOSOMAL OCULAR ALBINISM

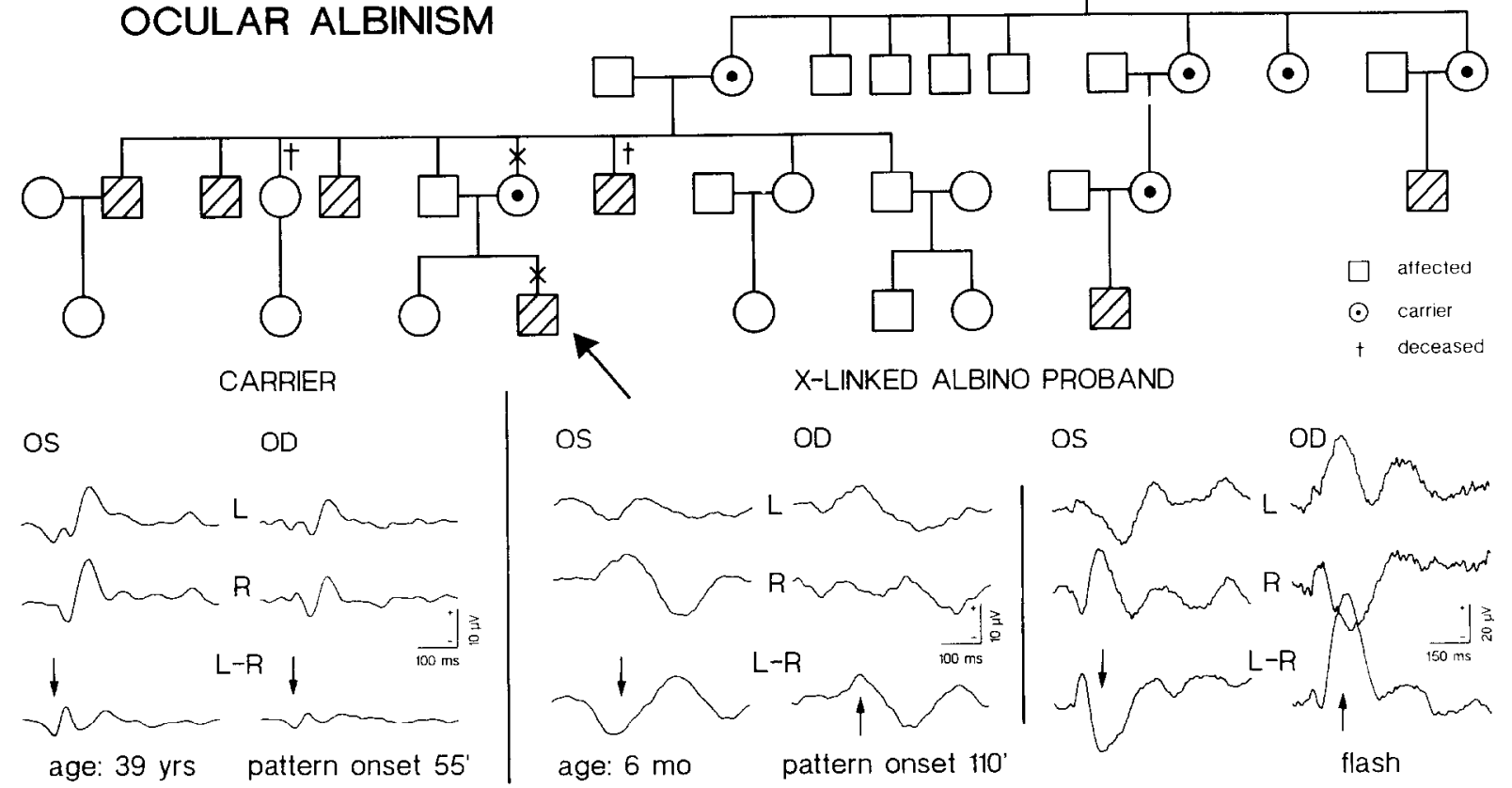

Fig. 16. VEP albino misrouting is not detected in an obligate heterozygote albino carrier (lower left). The potential distribution of monocular pattern onset VEPs in the albino carrier remains stable from left to right eye stimulation. In contrast. monocular pattern onset and luminance flash VEPs show the albino VEP misrouting profile. While contralateral albino VEP asymmetry is present in the pattern unset VEPs of the young proband, rather than showing component specificity, the whole positive peak appears to shift from right to left occiput following left and right eye stimulation, respectively. Asymmetry in the immature flash waveform is clear. The family pedigree across four generations, shows an X-chromosomal mode of inheritance. The proband is denoted by the arrow and the carrier, whose data are depicted, by an $\mathrm{X}$ (adapted from [1]).

VEP misrouting test which relies upon relative VEP topography, is essential for albino detection and differential diagnosis.

\section{Conclusions}

With strict adherence to non-invasive electruphysiological recording methods and test conditions specifically adapted for infants and young children, the VEP proves to be a sensitive and reliable diagnostic probe of visual function and dysfunction in paediatric ophthalmogenetics. Moreover, VEP assessment in the infant or child can readily provide accurate evaluation of the level of visual system maturation and with, follow-up studies, also the rate of growth. For VEP analysis, the electrophysiological response can be reduced to four basic parameters, amplitude, latency, waveform and topography. Determination of whether one or more variable falls within normal limits is dependent upon comparisons with age matched normal controls tested under the same conditions. Finally, the additional advantage of relying upon the VEP in the practice of ophthalmogenetics is that VEP testing can facilitate pedigree analysis by defining, for certain genetic disorders, affected family members.

\section{References}

[1] Apkarian, P. and Spekreijse, H. (1990) The use of the electroretinogram and visual evoked potentials in ophthalmogenetics. In: J.E. Desmedt (Ed.), Visual Evoked 
Potentials, Elsevier Science Publishers BV, Amsterdam, pp. 169-223.

[2] Van der Tweel, L.H. (1979) Pattern evoked potentials: facts and considerations. In: Proc. 16th Int. Soc. Clin. Electrophysiol. Vision Symp., Morioka, Japan, pp. 27-46.

[3] Apkarian, P. (1994) Visual evoked potential assessment of visual function in pediatric neuroophthalmology. In: D.M. Albert and F.A. Jakobeic (Eds.), Principles and Practice of Ophthalmology, Basic Sciences, W.B. Saunders \& Co., Philadelphia, PA, pp. 622-647.

[4] Rosenberg, M.I. and Jabhari, B. (1987) Nystagmus and visual evoked potentials. Neuro-Ophthalmology, 7: 133138.

[5] Dagnelie, G., de Vries, M.J., Maier, J. and Spekreijse, H. (1986) Pattern reversal stimuli: motion or contrast? Doc. Ophthalmol., 61: 343-349.

[6] Estévez, O. and Spekreijse, II. (1974) Relationship between pattern appearance-disappearance and pattern reversal responses. Exp, Brain Res., 19: 233- 238.

[7] Spekreijse, H., Dagnelie, G., Maier, J. and Regan, D. (1985) Flicker and movement constituents of the pattern reversal response. Vision Res., 25: 1297-1304.

[8] Arlt, A. and Zangemeister, W.H. (1990) Influence of slow eye movements and nystagmus on pattern induced visual evoked potentials. Neuro-Ophthalmology, 10: 241 251.

[9] Apkarian, P. (1993) Temporal frequency responsivity shows multiple maturational phases: state-dependent visual evoked potential luminance flicker fusion from birth to 9 months. Vis. Neurosci., 10: 1007-1018.

[10] Apkarian, P., Eckhardt, P.G. and van Schooneveld, M.J. (1991) Detection of optic pathway misrouting in the human albino neonate. Neuropediatrics, 22: 211-215.

[11] De Vries Khoe, L.H. and Spekreijse, H. (1982) Matura tion of luminance and pattern EPs in man. Doc. Ophthaimol., 31: 461-475.

[12] Apkarian, P., van Veendendaal, W. and Spekreijse, $H$. (1986) Measurement of visual acuity in infants and young children by visual evoked potentials. Doc. Ophthalmol., 45: $168-189$.

[13] Bleeker-Wagemakers, E.M. (1984) Visual impairment in the mentally retarded. In: J. Dobbing, A.D.B. Clarke, J.A. Corbett, J. Hogg and R.O. Robinson (Eds.), Scientific Studies in Mental Retardation, The Royal Society of Medicine and The Macmillan Press Ltd., London, pp. 299-321.

[14] Harter, M.R. and White, C.T. (1968) Effects of contour sharpness and check-size on visually evoked cortical potentials. Vis. Res. 8: 701-711.

[15] Millodot, M. (1977) The use of visual evoked potentials in optometry. In: J.E. Desmedt (Ed.), Visual Evoked Potentials In Man: New Developments, Clarendon Press, Oxford, pp. 401-409.

[16] Millodot, M. and Riggs, L.A. (1970) Refraction determined electrophysiologically. Arch. Ophthalmol., 84: 272-278.

[17] Millodot, M. and Newton, I. (1981) VEP measurement of the amplitude of accommodation. Br. J. Ophthalmol., 65: 294-298.

[18] Apkarian, P. (1992) A practical approach to albino diagnosis. VEP misrouting across the age span. Ophthalm. Paediat. Genet., 13: 77-88.

[19] Kinnear, P.E., Jay, B. and Witkop, C.J., Jr. (1985) Albinism. Surv. Ophthalmol., 30: 75-101.

[20] Guillery, R.W., Okoro, A.N. and Witkop, C.J., Jr, (1975) Abnormal visual pathways in the brain of a human al. bino. Brain Res., 96: 373-377.

[21] Apkarian, P., Reits, D., Spekreijse, H. and van Dorp, D. (1983) A decisive electrophysiological test for human albinism. Electroenceph. Clin. Neurophysiol., 55: 513531.

[22] Shallo-Hoffmann, J. and Apkarian, P. (1993) Visual evoked response asymmetry only in the albino member of a family with congenital nystagmus. Invest. Ophthalmol. Vis. Sci., 34: 682-689. 